\title{
Reply to Dr. Goodwin
}

To the Editor:

Dr. Goodwin misinterprets our emphasis on the importance of the mitogen dose dependency of the indomethacin (IND) effect on lymphocyte functions in evaluating such experimental data. Our major concern stems from the fact that the optimal concentration of mitogen is a variable entity and depends upon culture conditions as well as the individual donor source (1-3). Contrary to the statement that all their studies on prostaglandin (PG) regulation of immune functions used suboptimal mitogen concentrations, Dr. Goodwin and his colleagues have previously claimed that "all further experiments were performed at optimal concentrations of the mitogen" (4). A similar statement was made in one of their other publications (5). Their experimental evidence implicating a role for endogenous PGs on the immune response in human physiology (5) and pathology (4) was based on studies employing a single mitogen concentration. In our paper (3) we discussed how the use of a single mitogen concentration could give rise to a misleading interpretation.

We would like to point out that our skepticism on the effect of endogenous PGs on the immune response was based on several observations. In particular, we observed that exogenously added PGs suppress mitogenic responses at all mitogen concentrations tested including the optimal mitogenic dose, whereas IND enhances the transformation response only at suboptimal mitogen doses and fails to cause enhancement at the optimal concentration of the mitogen. If IND were acting through the inhibition of PG synthesis one would expect an enhancement of the transformation response even at an optimal concentration of the mitogen, i.e., the reciprocal of the PG effect. These observations lead us to suggest that the effect of IND on the mitogenic response is mediated through some other pharmacological activity, such as its effect on cyclic AMP-dependent protein kinase (6).

\section{K. Murali Krishna Rao Stanley A. Schwartz Department of Pediatrics, University of Michigan, Ann Arbor, Michigan 48109}

\section{REFERENCES}

1. Cunningham-Rundles, S., Hansen, J. A., and Dupont, B., In "Clinical Immunobiology" (F. H. Bach and R. A. Good, Eds.), Vol. 3, pp. 151-194. Academic Press, New York, 1976.

2. Goodwin, J. S., Messner, R. P., and Peake, G. T., J. Clin. Invest. 62, 753, 1978.

3. Rao, K. M. K., Schwartz, S. A., and Good, R. A., Cell. Immunol. 48, 155, 1979.

4. Goodwin, J. S., Messner, R. P., Bankhurst, A. D., Peake, G. T., Saiki, J. H., and Williams, R. C., Jr., N. Engl. J. Med. 297, 963, 1977.

5. Goodwin, J. S., Bankhurst, A. D., and Messner, R. P., J. Exp. Med. 146, 1719, 1977.

6. Kantor, H. S., and Hampton, M., Nature (London) 276, 861, 1978. 